\title{
Computer Simulation of Protein-Protein Association in Photosynthesis
}

\author{
I.B. Kovalenko ${ }^{1 *}$, A.M. Abaturova ${ }^{1}$, A.N. Diakonova ${ }^{1}$, O.S. Knyazeva ${ }^{2}$, \\ D.M. Ustinin ${ }^{1}$, S.S. Khruschev ${ }^{1}$, G.Yu. Riznichenko ${ }^{1}$ and A.B. Rubin ${ }^{1}$ \\ ${ }^{1}$ Biological faculty, Lomonosov Moscow State University, \\ Leninskye Gory, Moscow 119992, Russia \\ ${ }^{2}$ Physical faculty, Lomonosov Moscow State University, \\ Leninskye Gory, Moscow 119992, Russia
}

\begin{abstract}
The paper is devoted to the method of computer simulation of protein interactions taking part in photosynthetic electron transport reactions. Using this method we have studied kinetic characteristics of protein-protein complex formation for four pairs of proteins involved in photosynthesis at a variety of ionic strength values. Computer simulations describe non-monotonic dependences of complex formation rates on the ionic strength as the result of long-range electrostatic interactions. Calculations confirm that the decrease in the association second order rate constant at low values of the ionic strength is caused by the protein pairs spending more time in "wrong" orientations which do not satisfy the docking conditions and so do not form the final complex capable of the electron transfer.
\end{abstract}

Key words: Brownian dynamics, computer simulation, protein, photosynthesis, electrostatic interaction

AMS subject classification: $92 \mathrm{C} 05,92 \mathrm{C} 40,92-08$

\section{Introduction}

Many biochemical processes are associated with protein-protein complex formation. In photosynthesis of higher plants the electron flow passes through mobile electron carrier proteins plas-

*Corresponding author. E-mail: ikovalenko78@gmail.com 
tocyanin $(\mathrm{Pc})$ and ferredoxin $(\mathrm{Fd})$. Pc, a small copper protein, makes transient complexes with cytochrome $f$ of cytochrome $b f$ complex $(\mathrm{Cyt} f)$ and with the luminal part of photosystem I (PSI) shuttling electrons from cytochrome $b f$ complex to P700, the reaction center of PSI [10] in the luminal space of chloroplasts. Fd diffuses in the chloroplasts stromal space and transfers electrons from PSI to ferredoxin-NADP ${ }^{+}$reductase (FNR), forming transient complexes with the stromal part of PSI and FNR.

Interaction process of an electron carrier protein with a multienzyme complex takes place in four steps [20]: (1) a small protein moves to the complex due to diffusion and electrostatic attraction; (2) interaction between separate parts of the molecules determined mainly by electric charge configuration, spatial orientation of the mobile molecules coupled to the formation of an initial predocking complex; (3) final binding and formation of an active complex capable of an electron transfer; and (4) the electron transfer process. At different steps of complex formation various factors play different roles. Diffusion of protein molecules, long-range electrostatic interactions, and molecules geometric shapes play a key role in the formation of the initial predocking complex. In their turn, Van-der-Waals and hydrophobic interactions, H-bonds and molecular flexibility in the protein-protein interface take important place in the transformation of the initial complex into the final complex.

Long-range electrostatic interactions significantly accelerate the process of precomplex formation and thereby make it much more effective. It is widely recognized that electrostatic interactions play a crucial role in the binding of Pc and Fd to their reaction partners [9, 28]: they govern the diffusion of a mobile protein to its docking sites at protein complexes orienting a mobile molecule in the electrostatic field and thus influencing an association rate.

Brownian dynamics (BD) models are usually used to simulate protein-protein interaction kinetics. In BD simulations the interaction of two individual proteins is investigated in solution, the proteins are treated as rigid or semi-rigid bodies, their geometric shape is considered at the atomic resolution, and electrostatic interactions of the proteins are presented in great details. There are BD models for the association of a pair of individual molecules Pc and Cyt $f[23,25,32]$. Currently there are no BD models of Fd-FNR reaction, although a model of association between ferredoxin and hydrogenase from Chlamydomonas reinhardtii has been presented recently [19]. These BD models simulate the complex formation of two individual protein molecules in solution. Reactions between pigment-protein complex PSI and mobile carrier proteins Pc and Fd have not been studied by BD approach yet.

Recently, we have developed a new BD-based method for multiparticle 3D computer simulation of simultaneous diffusion and interactions of several hundreds of proteins. Our approach makes it possible to simulate reaction kinetics and obtain reaction time courses directly from the computer simulation. This method was used to study Pc-Cyt $f$ complex formation for wild type (wt) and mutant Pc at a variety of ionic strength values in solution [14] and in the chloroplast thylakoid lumen [15]. The method was further developed to take into account the electrostatic interactions of proteins with the thylakoid membrane in the thylakoid lumen [13]. The method was applied for studying of electrostatic interactions effect on the rates of complex formation between PSI and mobile carrier proteins Pc and Fd at various ionic strength values to explain the experimentally observed non-monotonic dependences of the association second-order rate constant on 
the ionic strength for Pc-PSI and Fd-PSI reactions [17]. This approach was also used to study kinetic characteristics of Fd and FNR complex formation in solution [18]. Besides, this method was applied for prediction of binding areas and possible complex structures for several pairs of test proteins [16]. The results are summarized in reviews [25, 26, 27]. This review presents the results of our works on the simulation of protein-protein complex formation rate constant dependence on the ionic strength.

\section{The computational method}

The method is developed for simulation of the initial predocking complex formation, where diffusion of protein molecules and electrostatic interactions play the key role, so it does not describe complex formation in detail (such as hydrophobic interactions and conformational changes of proteins, which may be important for the final complex formation). In addition to capabilities of the BD method, our approach allows also to obtain by computer simulations the time curves of protein-protein complex formation, which can be compared with experimental time curves for different protein concentrations, ionic strength and $\mathrm{pH}$ values for the wild type (wt) and mutant forms of proteins.

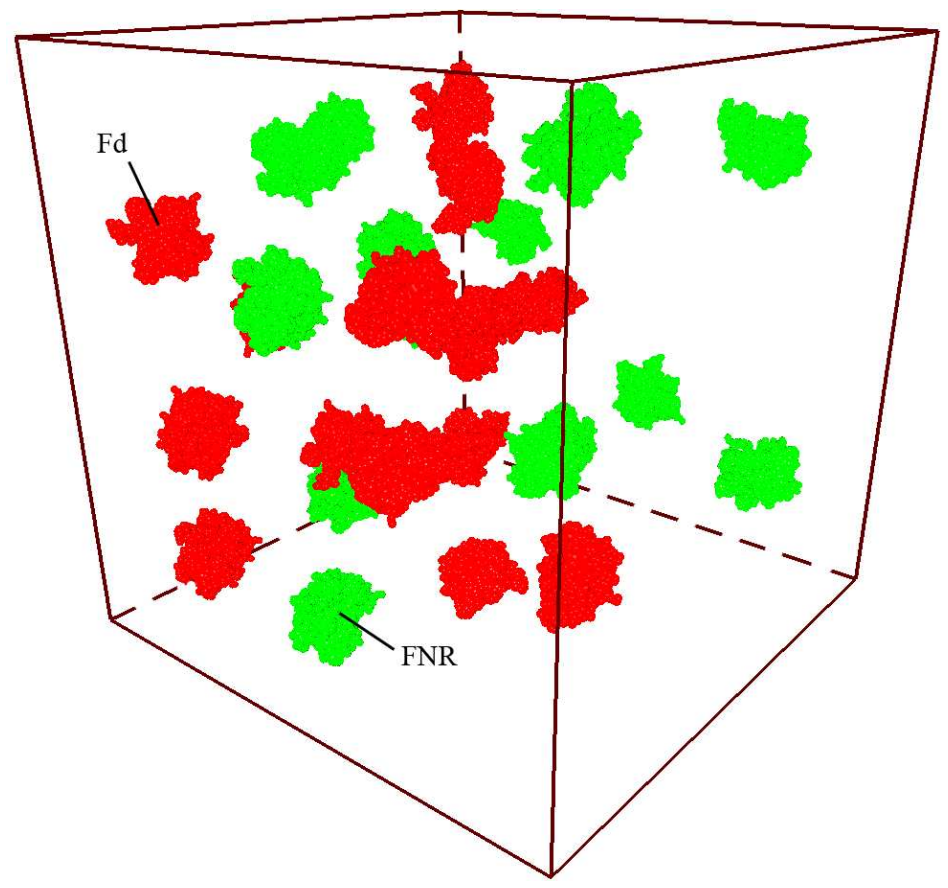

Figure 1: Visualization of computer simulation model scene with proteins Fd and FNR. 


\subsection{Simulation of protein diffusion}

Each protein molecule is represented by a 3D rigid body. The model scene is a parallelepiped with mirror boundary conditions. Protein molecules can be mobile or fixed at their initial positions. All molecules are considered to be mobile when modelling reaction in solution (see Fig. 1), membrane proteins are fixed along two opposite sides of the parallelepiped when the scene represents the chloroplast thylakoid lumen (see Fig. 4). In the process of simulation the mobile protein molecules diffuse in the reaction volume under the action of the random Brownian force and electrostatic force. As shown in [4], the Langevin equation can be used for the mathematical description of such process. The Langevin equation for translational motion has the following form:

$$
\xi_{\operatorname{tr}}^{x} \frac{d x}{d t}=f_{x}(t)+F_{x}
$$

where $x$ is a coordinate, $\xi_{\mathrm{tr}}^{x}$ is a viscous friction coefficient at $x, f_{x}(t)$ and $F_{x}$ are projections of the random Brownian force and electrostatic force onto the $x$ axis, respectively. The random Brownian force has a normal distribution with zero mean value and dispersion $2 k T \xi_{\mathrm{tr}}^{x} / \triangle t$. Here $k$ is the Boltzmann constant, $T$ is temperature, $\triangle t$ is time step, $F_{x}=-q d \varphi / d x, q$ is the charge, $\varphi$ is the electrostatic potential.

The Langevin equation for rotational motion:

$$
\xi_{\text {rot }}^{x} \frac{d \alpha}{d t}=m_{x}(t)+M_{x}
$$

where $\alpha$ is the angle of rotation, $\xi_{\text {rot }}^{x}$ is the viscous friction coefficient for rotational motion around $x$ axis, $m_{x}(t)$ and $M_{x}$ are the moments of the random Brownian force and electrostatic force relative to the axis $x$, respectively. In hydrodynamic calculations protein molecules were presented as prolate spheroids with larger axis coinciding with axis of molecule minimum inertia and having the same moment of inertia. Viscous friction coefficients for spheroids were calculated using formulae from [1]. Dynamic viscosity of medium was considered as equal to that of water $\left(1 \cdot 10^{-3} \mathrm{~kg} \mathrm{~m}^{-1} \mathrm{~s}^{-1}\right.$ at $\left.293 \mathrm{~K}\right)$. For the description of each object position we have chosen the Cartesian coordinate system with mirror boundary conditions at the edges (the scene coordinate system). Every object is assigned to its local Cartesian coordinate system, which coincides with the respective spheroid axes. Random numbers were produced by the LEcuyer random number generator [22]. Time step for numerical integration of Langevin equation (100 ps) was chosen so that average displacement of molecules during one step was about the size of electrostatic potential grid (2 Å, see Subsection 2.2).

To simulate objects collisions, their shapes are described with sets of spheres so, that a deviation of the protein surface represented by the calculated spheres from the real protein atomic surface is less than $2 \AA$ (251 spheres for Anabaena Fd, 829 for FNR, 261 for Pc, 363 for spinach Fd and 3262 for PSI). Geometric representation of the molecular surface by a number of spheres is realistic, in this case corresponding calculations are less time consuming than calculations of each atom overlap. 


\subsection{Simulation of electrostatic interactions}

Electrostatic interactions play an important role in protein-protein complex formation. Partial charges of the proteins produce heterogeneous electrostatic field. The electrostatic potential rapidly decreases with the distance from the protein surface due to screening by polar water molecules. When a protein is far from other proteins, it moves under the action of the Brownian force but not the electrostatic force, and hence its motion is determined only by the Brownian diffusion. As the protein comes closer to other proteins, its motion is governed by the electrostatic field of the latter so that a favorable position for the initial complex formation can be reached. In the simulation the electrostatic interactions are taken into consideration only when the distance between two protein surfaces is less than $50 \AA$ because electrostatic interactions are very weak at greater distances. The magnitude of electrostatic field at these and greater distances from the protein surface is considered to be zero.

The electrostatic force acting on the $i$-th mobile molecule is calculated as

$$
\vec{F}^{i}=\sum_{i \neq j} \vec{F}_{i j}+\vec{F}_{\mathrm{env}}^{i}
$$

where $\vec{F}_{i j}$ is a force of interaction between mobile proteins $i$ and $j, \vec{F}_{\text {env }}^{i}$ is a force acting on protein $i$ by the electrostatic field of the membrane and membrane proteins immobile charges and solution ions. To calculate this force, the electrostatic potential was determined using the Poisson-Boltzmann equation [7, 31] that describes an electric field around the immobile charges with account of free ions:

$$
\nabla(\varepsilon(\vec{r}) \nabla \varphi(\vec{r}))=-\frac{1}{\varepsilon_{0}}\left(\rho_{\text {ions }}(\vec{r})+\rho_{\text {prot }}(\vec{r})+\rho_{\text {memb }}(\vec{r})\right),
$$

where $\varepsilon_{0}$ is the electric constant; $\rho(\vec{r}), \varepsilon(\vec{r})$ and $\varphi(\vec{r})$ are the local values of bulk charge density, permittivity and electrostatic potential, respectively.

Free ions are distributed homogeneously in solution, but this is not so directly near the protein and membrane surfaces. Disregarding the interaction between free ions, their distribution in the electrostatic field of immobile charges with potential $\varphi(\vec{r})$ obeys the Boltzmann law:

$$
c_{i}=c_{i}^{\mathrm{bulk}} \exp \left(-W_{i} / k T\right)
$$

where $c_{i}^{\text {bulk }}$ is the bulk ion concentration, $W_{i}=z_{i} e_{0} \varphi(\vec{r})$ is the work of carrying the ion from the bulk to the vicinity of point $(\vec{r})=(x, y, z)$. The density of ions is calculated as

$$
\rho_{\text {ions }}(\vec{r})=\sum_{i} c_{i} z_{i} e_{0}
$$

where $z_{i}$ is charge number, $e_{0}$ is electron charge, $c_{i}$ are local ion concentrations. Thus

$$
\rho_{\text {ions }}(\vec{r})=\sum_{i} c_{i}^{\text {bulk }} z_{i} e_{0} \exp \left(\frac{-z_{i} e_{0} \varphi(\vec{r})}{k T}\right) \approx 2 \mathrm{I} e_{0}^{2} \frac{\varphi(\vec{r})}{k T},
$$


where I is the ionic strength, $\mathrm{M}$.

The resulting equation is solved by iteration. To do this, the entire modelling region is partitioned into cubic $2 \AA$-edge cells. This size corresponds to average accuracy of NMR/X-ray PDB structures. For this reason we choose a 3D rectangular region around every protein, set up a rectangular grid in the region, and map the charges, dielectric constants, ionic strength to the grid. The dielectric constant for the cells inside the protein was assigned a value of 2 [6], inside the solution it is 80 , at the boundary protein-solution it is 40 , and the value of the ionic strength inside the protein was assigned zero. The potential in every such cell is calculated by the iterative formula using the values obtained at the preceding step in six neighboring cells [31]:

$$
\varphi_{i, j, k}^{n}=\frac{\sum_{m=1}^{6} \frac{\varepsilon_{m}+\varepsilon_{i, j, k}}{2} \varphi_{m}^{n-1}+\frac{1}{\varepsilon_{0} h}\left(q_{i, j, k}^{\text {prot }+ \text { memb }}\right)}{\sum_{m=1}^{6} \frac{\varepsilon_{m}+\varepsilon_{i, j, k}}{2}+\varkappa h^{2}},
$$

where $\varkappa=2 \mathrm{I} e_{0}^{2} /\left(\varepsilon_{0} k T\right) ; h$ is a grid step; $i, j, k$ are the numbers of a cell in 3D volume; $\varphi_{i, j, k}^{n}$ is the potential at the $n$-th step in point $i, j, k$, with summation over six neighboring cells; $\varepsilon_{m}$ and $\varphi_{m}^{n-1}$ are permittivity and potential at the preceding $n-1$ step in one of the cells neighboring $i, j, k$; and $q^{\text {prot+memb }}$ is a sum of protein and membrane charges included in the cell $i, j, k$.

To set the boundary conditions, the potential is equated to zero at a distance $>50 \AA$ away from the membrane surface because of the screening effect of water. The Poisson-Boltzmann equation for mobile proteins is solved analogously, zero boundary conditions are set at $50 \AA$ away from the protein surface. The formal charges on the proteins were calculated as described in [5]. The pKa values of the amino acid residues were taken from [3]. The solution $\mathrm{pH}$ is taken into account in the Henderson-Hasselbalch equation to calculate the charges on amino acids atoms, for all of the runs $\mathrm{pH}$ is 7 .

\subsection{Simulation of complex formation}

For good approximation, the preliminary complexes are formed by rigid-body association [12]. However, molecular flexibility in the protein-protein interface and hydrophobic interactions of proteins may be important for the transformation of the initial complex into the final complex. Our method does not explicitly simulate a transformation of the initial encounter complex into the final complex. Instead, this transformation is implicitly taken into account by introducing the probability $p$ of final complex formation.

When two protein molecules come closer to each other within the docking distance during the diffusion process, the proteins are considered to form an initial predocking (encounter) complex. The docking distance is a model parameter defined as a distance between particular atoms involved in complex formation. Usually, these atoms are proteins redox centers (cofactors), but for some protein pairs (for example, plastocyanin-cytochrome $f$ ) we additionally measure distances between contacting amino acid residues in order to reduce the number of possible initial complexes [14].

This initial complex transforms into the final complex with probability $p$, which is also a model parameter. So, all the possible orientations of the proteins within the encounter complex are con- 
sidered to form the final complex with probability $p$. The accidental nature of the final complex formation in the computer algorithm at the given $p$ value at each time step is implemented by a random number choice from the interval $[0 ; 1]$. If the generated number is less than the given docking probability $p$, it means that the complex formation at this time step occurs. If the generated number is greater than the given docking probability $p$, the program proceeds to the next step. Provided the proteins still satisfy the docking conditions, the described procedure is repeated. If three protein molecules come closer to each other, their interactions are handled by pairs subsequently.

By calculating directly the number of complexes formed at each time step and plotting it versus time, we obtain a complex formation kinetic curve (see Fig. 2). We can compare obtained kinetic

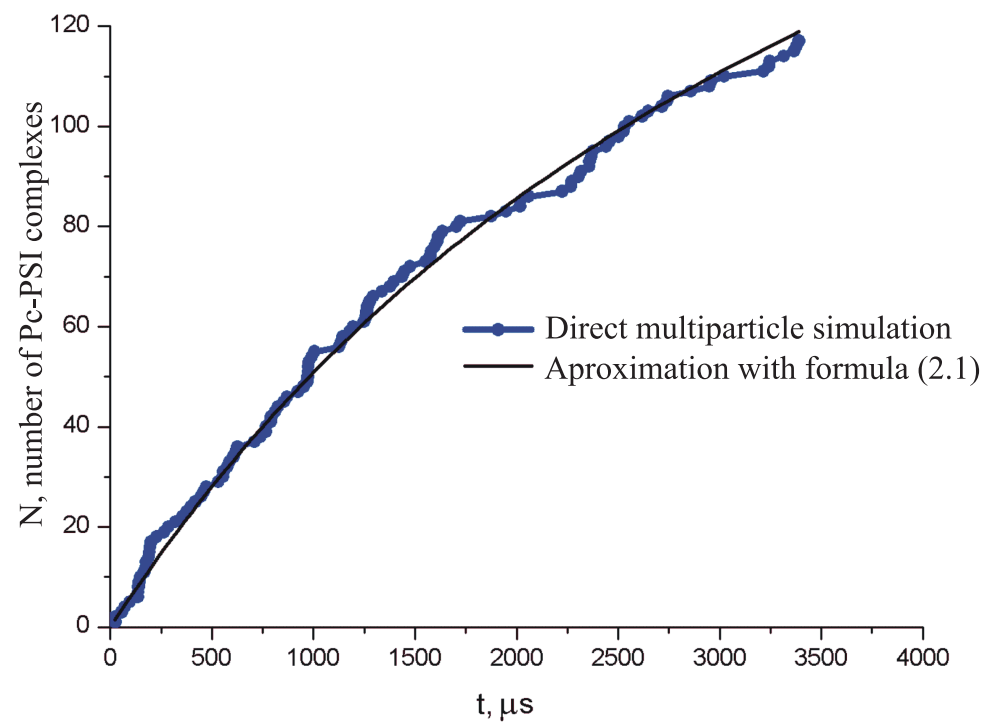

Figure 2: Calculated kinetic curve of Pc-PSI complex formation and its approximation by the mass action law (2.1), $N_{0}=270$ is the total number of Pc molecules, $k=3.9 \cdot 10^{8} \mathrm{M}^{-1} \cdot \mathrm{s}^{-1}$ is a calculated association second-order rate constant. The curve was calculated with the following parameter values: $\mathrm{I}=100 \mathrm{mM}, p=1, r=6 \AA$ (the distance between Asp44 on Pc and Lys23 of psaF on PSI). The figure is reprinted from [17] with permission from Elsevier.

curve with the kinetic curves which are registered experimentally, or we can use calculated curve as a source of information about rate constants of corresponding biochemical reaction. To do this, we consider a simple kinetic scheme of a second-order reaction

$$
A+B \stackrel{k}{\longrightarrow} A B
$$

where $k$ is the second-order association rate constant, $A$ denotes the first protein, $B$ denotes the second protein. This kinetic scheme is undoubtedly a strong simplification. The goal of such scheme consideration is to estimate the rate constant of protein-protein complex formation in the assumption that the reaction is bimolecular and compare the obtained values with experimental data which are usually presented as bimolecular rate constants. Bimolecular kinetic scheme matches the calculated kinetic curves well, if the solution concentration is not very high. 
For the ideal solution, a bimolecular reaction rate is proportional to the product of reacting species concentrations (the mass action law). Thus, if the initial concentrations of reagents are the same and equal to $N_{0}$, then the kinetic equation of the complex formation has the following form:

$$
\frac{d C}{d t}=k\left(N_{0}-C\right)^{2}
$$

where $C$ is the number of complexes formed. Integrating of this equation will give

$$
C(t)=N_{0}-\frac{N_{0}}{N_{0} k t+1} .
$$

We see that in case of equal initial concentrations of reagents, the time curve is a hyperbola. The rate constant can be determined by fitting the calculated kinetic curve from (2.1).

\section{Results and discussion}

\subsection{Electrostatic potential field of Fd, PSI and Pc}

Figure 3 displays the two ways of electrostatic potential visualization. Equipotential electrostatic surfaces show the distribution of the electrostatic field potential in the area surrounding the protein molecule. Surface electrostatic potential shows the charge distribution on the surface of the protein molecule. The protein surfaces were built according to the Connolly algorithm [2]. Although both proteins have negative net charge ( -2 for $\mathrm{Cyt} f$ and -8 for $\mathrm{Pc}$ at $\mathrm{pH} 7$ ), the binding area on Cyt $f$ is positively charged, while the binding area on Pc is negatively charged (see Fig. 3), which results in molecules attraction and proper orientation and promotes the complex formation.

Figure 4 shows the luminal space between two photosynthetic membranes. Cyt $f$ molecules are shown in green. The membrane creates a negative electrostatic field, its intensity is maximum near the membrane surface and declines towards a lumen middle. The equipotential surfaces calculated by the Poisson-Boltzmann equation for the membranes and four Cyt $f$ molecules are shown in red for -6.5 and blue for $+6.5 \mathrm{mV}$.

\subsection{Second order rate constant dependence on the ionic strength}

Electrically charged amino acid residues on the protein surface generate heterogeneous electrostatic field which is screened by polar water molecules and ions in the solution. Increasing ionic strength leads to a decrease in proteins electrostatic interaction due to a protein electric charges screening by salt ions. The character of the dependence of the rate constant of the protein binding on the ionic strength shows the role of electrostatic interactions in protein binding: if the rate constant decreases monotonically with increasing ionic strength, it means that electrostatic interactions between reagents favor protein binding. The dependence of the rate constant of protein-protein association on the ionic strength can be monophasic (the rate constant either decreases or increases 

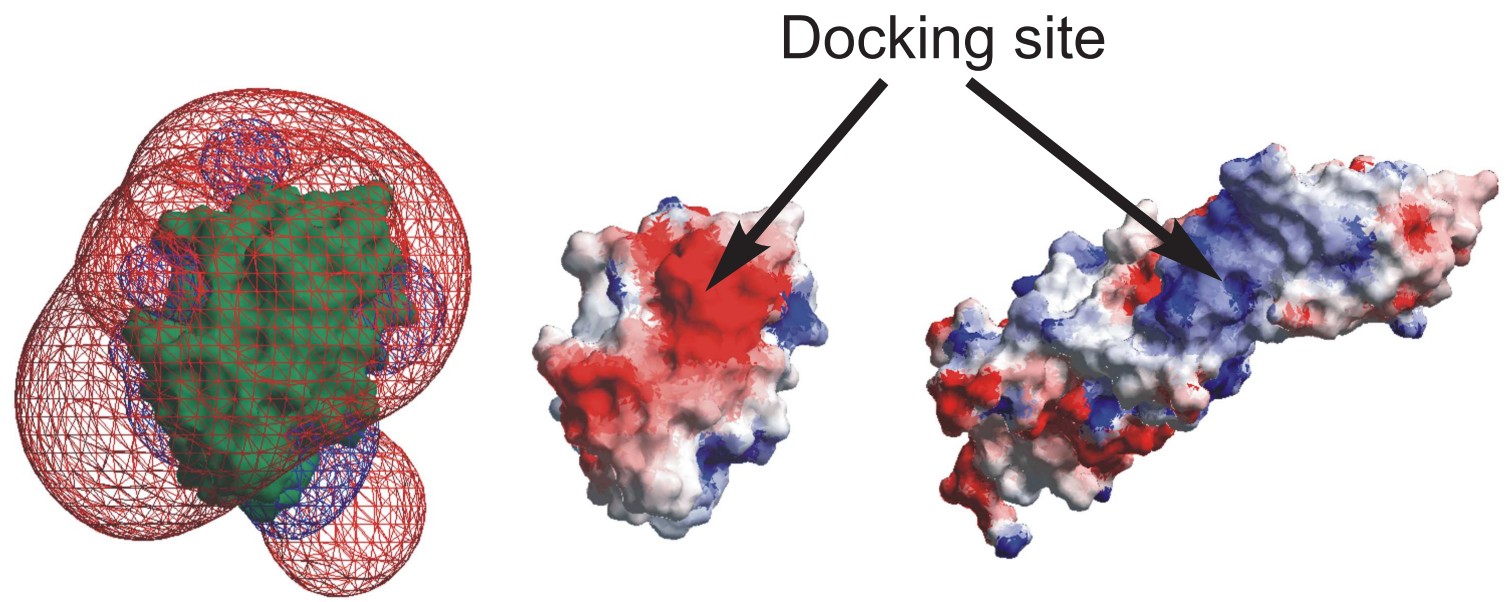

Figure 3: The two ways of electrostatic potential visualization: left - equipotential surfaces +6.5 $\mathrm{mV}$ (blue) and $-6.5 \mathrm{mV}$ (red) of Pc; middle and right — surface electrostatic potential of Pc and Cyt $f$, respectively. The surfaces were built according to the Connolly algorithm [2] and the potential value at every point is shown by blue (positive potential) and red (negative potential) color.

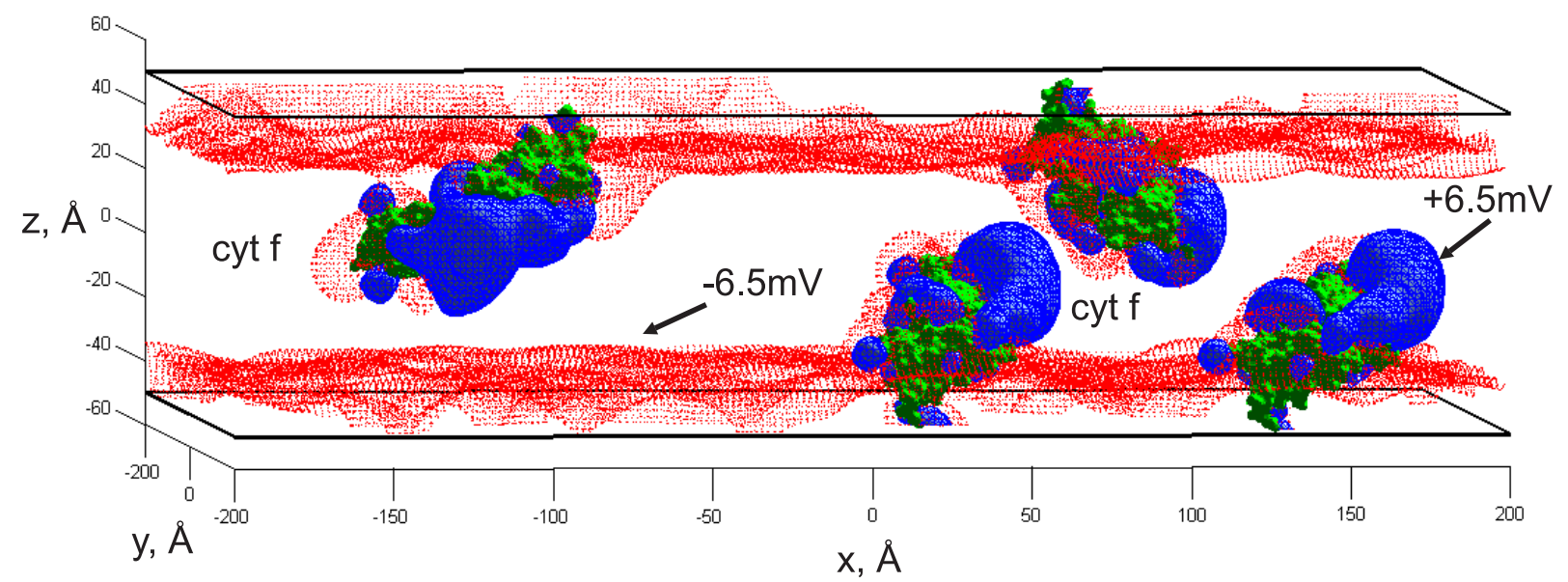

Figure 4: Equipotential surfaces $(+6.5 \mathrm{mV}$ red and $-6.5 \mathrm{mV}$ blue $)$ of a photosynthetic membrane and Cyt $f, \mathrm{pH}=7, \mathrm{I}=100 \mathrm{mM}$, membrane charge density $\sigma=-23.36 \mathrm{mC} / \mathrm{m}^{2}$. 
monotonically with the increasing ionic strength), biphasic (bell-shaped), and independent from the ionic strength.

Attraction between the negatively charged regions of Pc and the positive ones of Cyt $f$ assists in their closing to form a complex. Calculated second-order rate constants for Pc-Cyt $f$ complex formation at different membrane charge densities $\sigma$ and different ionic strength values are presented in Fig. 5. The ionic strength dependences for all membrane charge densities values are bell-shaped: the rate constant at low ionic strength values increases with the increasing ionic strength, reaches its maximum value at $30 \mathrm{mM}$ ionic strength, and decreases at higher salt concentrations. The decreasing parts of the curves at high ionic strength are the result of protein charges shielding by solution counterions which weaken the electrostatic interactions between the proteins and, thus, lower the reaction rate. On the other hand, since electrostatic attraction is very strong at low ionic strength, the approach of two proteins can give rise to unproductive intermediates that do not meet the docking conditions and the proteins can remain "wrong" oriented for a long time that does not satisfy the docking conditions. The main hypothesis explaining the non-monotonic behavior consists in the formation of tight but nonproductive complexes due to the electrostatic interactions strength at low salt concentration $[21,30]$. Strong electrostatic attraction does not let the Brownian force break the "wrong" complex and try to make the productive complex again.

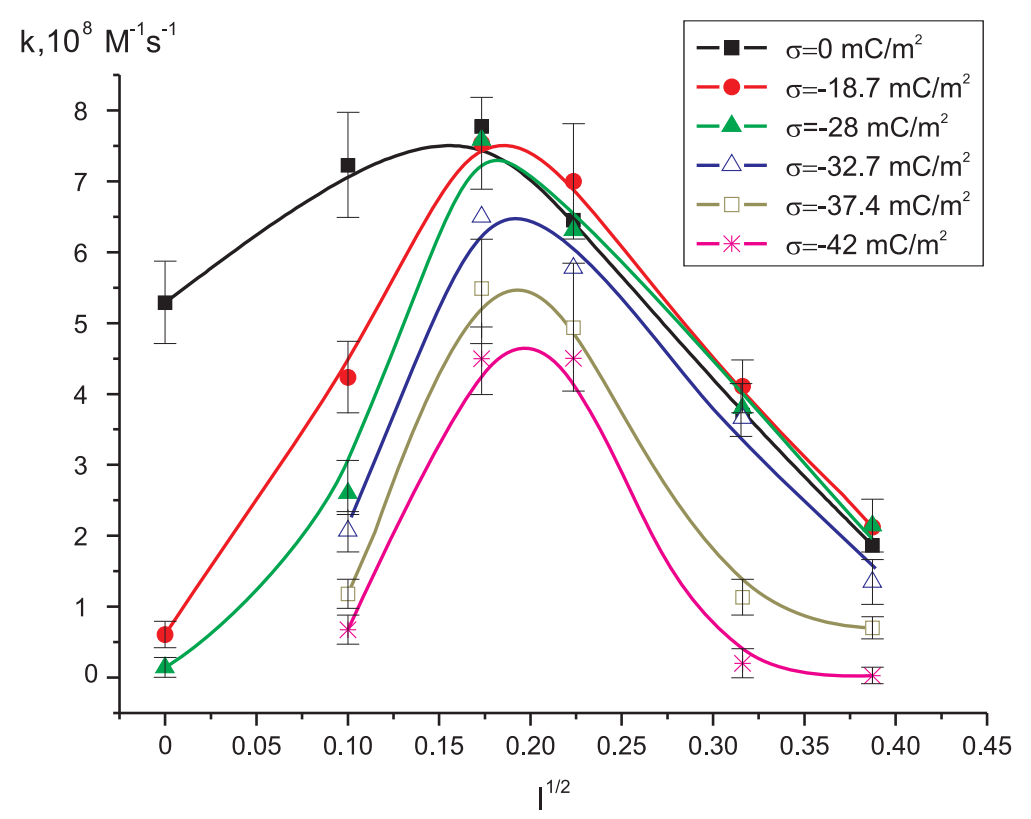

Figure 5: Dependence of the second-order rate constant of Pc-Cyt $f$ association on the square root of the ionic strength for different values of membrane charge density $\sigma$.

A photosynthetic membrane surface charge is another factor affecting the rate of $\mathrm{Pc}-\mathrm{Cyt} f$ complex formation in thylakoid lumen: its negative electrostatic field prevents the negatively charged Pc from approaching the membrane-bound $\mathrm{Cyt} f$, so the more negative is a membrane charge, the less is a rate constant of complex formation (see Fig. 5). The most negative charge density of a 
membrane $\sigma=-42 \mathrm{mC} / \mathrm{m}^{2}$ in the simulation corresponds to a membrane consisting only of lipids (about 17\% of membrane area is occupied by charged lipids). Ionic strength dependences for PcCyt $f$ reaction for various Pc mutants are presented in [14]. Spinach Pc and turnip Cyt $f$ molecular structures were taken from PDB ID 2PCF, docking distance values are given in [14], probability of final complex formation was taken as $p=0.01$.

Calculated dependencies of the second order rate constant value upon ionic strength for $\mathrm{Fd}-$ FNR reaction for different FNR mutants are presented in Fig. 6. It is clear from this figure that the wild type and FNRs with mutations Arg100Ala, Arg264Glu and Lys75Gln have a non-monotonic dependence on the ionic strength, while Lys138Glu, Arg16Glu and Lys72Glu show monotonic behavior. The reason for such difference in behavior is that the proteins with charge-reversal mutations in a neutral potential area (Lys138Glu, Arg16Glu and Lys72Glu) are largely impaired by electron transfer and even at low ionic strength values the electrostatic interactions are not strong enough to form nonproductive complexes and so the rate constants decrease monotonically with increasing ionic strength. The Lys75Gln mutant which is also largely impaired by electron transfer is not charge-reversal and electrostatic attraction to negatively charged Fd is still strong which can lead to a formation of non-productive complexes at low ionic strength values and non-monotonic ionic strength dependence. Such behavior was also obtained experimentally [11].

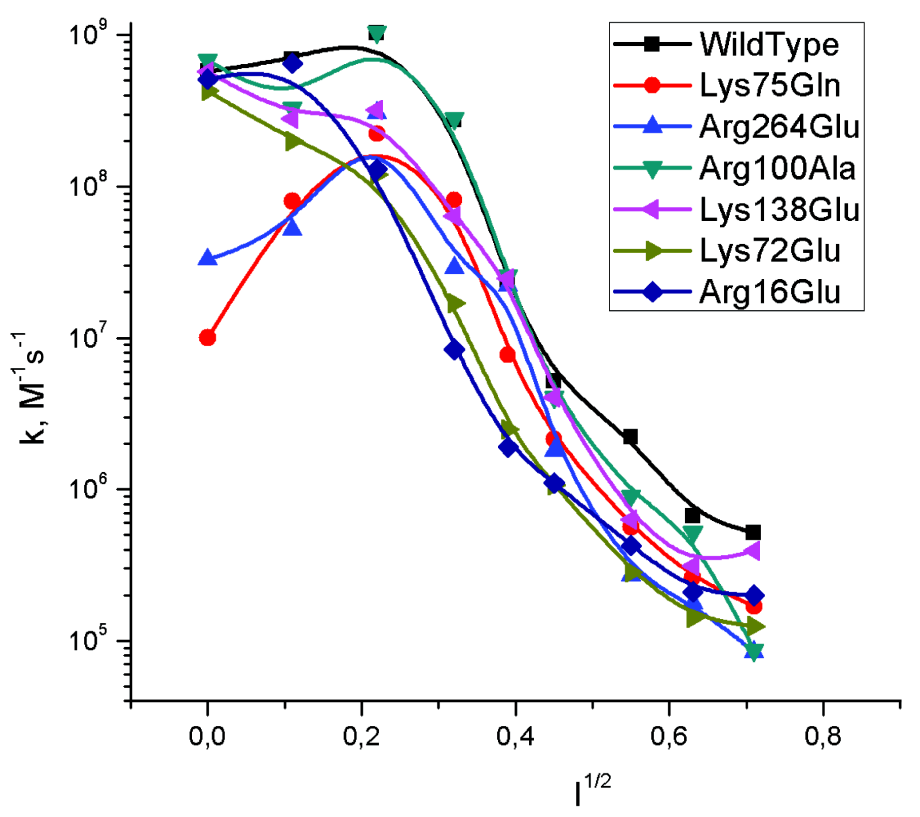

Figure 6: Second-order rate constant $\mathrm{k}$ dependence on the root square of the ionic strength I for the wild-type and mutant FNR and Fd: simulation results [18].

Anabaena Fd and FNR molecular structures were taken from PDB ID 1EWY. Docking distance (between [2Fe-2S] cluster on Fd and FAD on FNR) was taken as $18 \AA$ and probability of final Fd-FNR complex formation $p=0.003$. The calculated second-order rate constant dependences on the ionic strength for Pc-PSI and Fd-PSI reactions are presented in Figs. 7 and 8. For both reactions the dependences are bell-shaped: the rate constants at low ionic strength values increase 
with increasing ionic strength, reach their maximum values at $\mathrm{I}=30-50 \mathrm{mM}$, and then decrease at higher salt concentrations. The experimentally registered curves of observed rate constants show the same behavior [8, 29]. At ionic strength values lower than $30 \mathrm{mM}$ the electrostatic attraction is very strong which can prevent a proper orientation of mobile proteins $\mathrm{Pc}$ and $\mathrm{Fd}$ and make the mobile molecules remain "wrong" oriented for a long time that does not satisfy the docking conditions. At ionic strength values higher than $30 \mathrm{mM}$ the electrical charges on the proteins surfaces are shielded by counterions which weakens the electrostatic interactions between the proteins and lowers the reaction rate, and molecules don't remain "wrong" oriented for a long time.

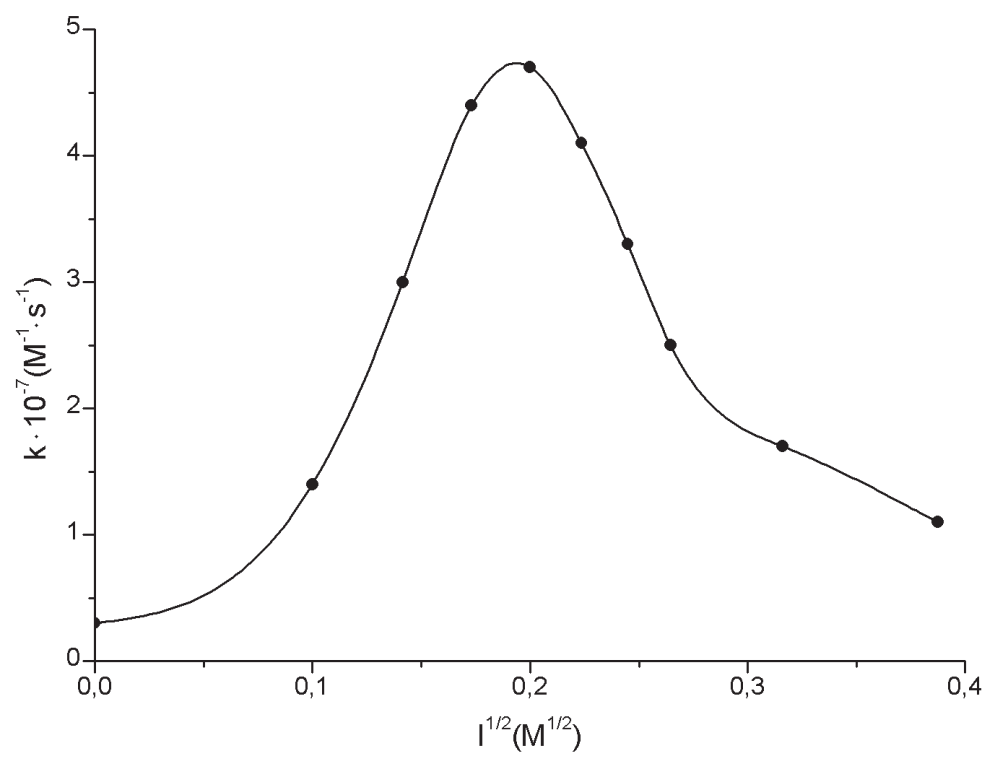

Figure 7: Calculated dependence of the association second order rate constant $k$ on the square root of the ionic strength I for Fd and PSI binding. The figure is reprinted from [17] with permission from Elsevier.

Spinach Pc structures were taken from PDB ID 2PCF, spinach Fd is from PDB ID 1A70, and higher plant (Pisum/Spinacia/Arabidopsis/Phaseolus) PSI with light harvesting complex I (LHCI) is from PDB ID 2O01. Model parameter values for Pc-PSI complex formation are $p=1$ and $r=6 \AA$ (the distance between OD1 atom of Asp44 on Pc and nitrogen atom of Lys23 of PsaF on PSI), for Fd-PSI complex formation are $p=1$ and $r=20.5 \AA$ (the distance between $\mathrm{Fe} 1$ atom of the $[2 \mathrm{Fe}-2 \mathrm{~S}]$ cluster on $\mathrm{Fd}$ and $\mathrm{Fe} 1$ atom of the $\mathrm{F}_{\mathrm{B}}$ cofactor on PSI). The multisubunit proteinpigment complexes of PSI with LHCI are fixed in the reaction volume, since molecular complex PSI with LHCI is much larger and heavier than Pc or Fd molecules, and so its diffusion coefficient is approximately five times less than for that of $\mathrm{Pc}$ or $\mathrm{Fd}$. 


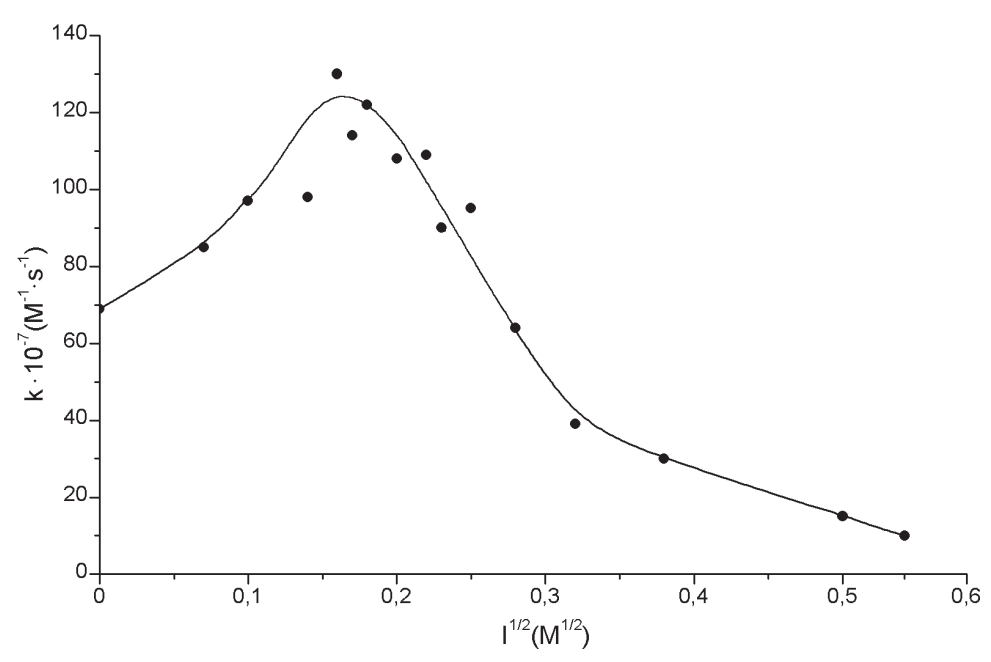

Figure 8: Calculated dependence of the association second order rate constant $k$ on the square root of the ionic strength I for Pc and PSI binding. The figure is reprinted from [17] with permission from Elsevier.

\section{Conclusion}

The simulation method presented in this paper can be applied for diffusion and functioning studying of many macromolecules that interact in the heterogeneous interior of subcellular systems. A characteristic feature and novelty of our method gives an opportunity to use it for studying of several protein molecules interaction simultaneously. It makes possible to simulate the formation of a large number of complexes taking place in solution or cell compartments and to monitor this process real-time kinetics.

The method allows to study the role of electrostatic interactions in protein complex formation. Though it does not clearly consider conformational changes and electron transport, it can help to reveal the effect of electrostatic potential changes upon the kinetic parameters of protein interaction and to evaluate the rate constant values of complex formation. For the Pc-Cyt $f$ and Fd-FNR complex formation in solution the simulated dependencies of the reaction rate constant on the ionic strength for different plastocyanin and FNR mutants are in good agreement with the experiments (see $[14,18]$ ). It indicates that for these reactions electrostatic forces play specific and very important role.

Our model reproduces the non-monotonic dependence of the association second order rate constant of protein binding on the ionic strength taking into account only electrostatic interactions $[13,14,15,16,17,18,25,26,27]$. Our calculations confirm the theory [21, 30] which supposes that a decrease in the rate constant at low values of the ionic strength is caused by the shift of equilibrium to an ensemble of complexes that are not optimal for the electron transfer. At low ionic strength protein molecules spend more time in "wrong" orientations, which do not satisfy the docking conditions (in the computer simulation) or do not form a final complex capable of electron 
transfer.

\section{Acknowledgements}

This work was supported by the grants P2219, N.Sh.-7885.2010.4 and RFBR 08-04-00354. Calculations were performed using Lomonosov Moscow State University supercomputer "Chebyshev".

\section{References}

[1] V.A. Bloomfield. Survey of biomolecular hydrodynamics. On-Line Biophysics Textbook: Separations and Hydrodynamics, 2000.

[2] M.L. Connolly. Analytical molecular surface calculation. J. Appl. Crystallogr., 16 (1983), $548-558$.

[3] R.M.C. Dawson, D.C. Elliott, W.H. Elliott, K.M. Jones. Data for biochemical research. Oxford Science Publications, OUP, Oxford, 1986.

[4] M. Doi, S.F. Edwards. The theory of polymer dynamics. Oxford University Press, New York, 1986.

[5] S.R. Durell, J.K. Labanowski, E.L. Gross. Modeling of the electrostatic potential field of plastocyanin. Arch. Biochem. Biophys., 277 (1990), 241-254.

[6] A.V. Finkelstein, O.B. Ptitsyn. Protein physics. A course of lectures. Academic Press, Amsterdam/Boston/London/New York/Oxford/Paris/San Diego/San Francisco/Singapore/Sydney/Tokyo, 2002.

[7] F. Fogolari, A. Brigo, H. Molinari. The Poisson-Boltzmann equation for biomolecular electrostatics: A tool for structural biology. J. Mol. Recognit., 15 (2002), 377-392.

[8] M. Hervas, M. De la Rosa, G. Tollin. A comparative laser-flash absorption spectroscopy study of algal plastocyanin and cytochrome c552 photooxidation by photosystem I particles from spinach. Eur. J. Biochem., 203 (1992), 115-120.

[9] M. Hippler, F. Drepper. Electron Transfer Between Photosystem I and Plastocyanin or Cytochrome c6, in Photosystem I: The Light-Driven Plastocyanin:Ferredoxin Oxidoreductase. J. (Ed. H. Golbeck). Springer, 2006, 499-513.

[10] A.B. Hope. Electron transfers amongst cytochrome f, plastocyanin and photosystem I: kinetics and mechanisms. Biochim. Biophys. Acta, 1456 (2000), 5-26. 
[11] J.K. Hurley, J.T. Hazzard, M. Martinez-Julvez, M. Medina, C. Gomez-Moreno, G. Tollin. Electrostatic forces involved in orienting Anabaena ferredoxin during binding to Anabaena ferredoxin:NADP+ reductase: site-specific mutagenesis, transient kinetic measurements, and electrostatic surface potentials. Protein Sci., 8 (1999), 1614-1622.

[12] J. Janin. Kinetics and thermodynamics of protein-protein interactions. Protein-protein recognition. (Ed. C. Kleanthous). Oxford University Press, Oxford, 2000, 1-32.

[13] O.S. Knyazeva, I.B. Kovalenko, A.M. Abaturova, G.Y. Riznichenko, E.A. Grachev, A.B. Rubin. Multiparticle computer simulation of plastocyanin diffusion and interaction with cytochrome $f$ in the electrostatic field of the thylakoid membrane. Biophysics, 55 (2010), No. 2, 221-227.

[14] I.B. Kovalenko, A.M. Abaturova, P.A. Gromov, D.M. Ustinin, E.A. Grachev, G.Y. Riznichenko, A.B. Rubin. Direct simulation of plastocyanin and cytochrome $f$ interactions in solution. Phys. Biol., 3 (2006), 121-129.

[15] I.B. Kovalenko, A.M. Abaturova, P.A. Gromov, D.M. Ustinin, G.Y. Riznichenko, E.A. Grachev, A.B. Rubin. Computer simulation of plastocyanin-cytochrome $f$ complex formation in the thylakoid lumen. Biophysics, 53 (2008), No. 2, 140-146.

[16] I.B. Kovalenko, A.M. Abaturova, G.Y. Riznichenko, A.B. Rubin. A novel approach to computer simulation of protein-protein complex formation. Dokl. Biochem. Biophys., 427 (2009), 215-217.

[17] I.B. Kovalenko, A.M. Abaturova, G.Y. Riznichenko, A.B. Rubin. Computer simulation of interaction of photosystem 1 with plastocyanin and ferredoxin. BioSystems, 103 (2010), 180187.

[18] I.B. Kovalenko, A.N. Diakonova, A.M. Abaturova, G.Y. Riznichenko, A.B. Rubin. Direct computer simulation of ferredoxin and FNR complex formation in solution. Phys. Biol., 7 (2010), No. 2, 026001.

[19] H. Long, C.H. Chang, P.W. King, M.L. Ghirardi, K. Kim. Brownian dynamics and molecular dynamics study of the association between hydrogenase and ferredoxin from Chlamydomonas reinhardtii. Biophys. J., 95 (2008), 3753-3766.

[20] F.S. Mathews, A.G. Mauk, G.R. Moore. Protein-protein complexes formed by electron transfer proteins, in Protein-Protein recognition. (Ed. C. Kleanthous). Oxford University Press, Oxford, 2000, 60-101.

[21] M. Medina, M. Hervas, J.A. Navarro, M.A. De la Rosa, C. Gomez-Moreno, G. Tollin. A laser flash absorption spectroscopy study of Anabaena sp. PCC 7119 flavodoxin photoreduction by photosystem I particles from spinach. FEBS, 313 (1992), No. 3, 239-242. 
[22] F. Panneton, P. L'Ecuyer. On the xorshift random number generators. ACM T. Model. Comput. Sci., 15 (2005), No. 4, 346-361.

[23] D.C. Pearson, E.L. Gross. Brownian dynamics study of the interaction between plastocyanin and cytochrome f. Biophys. J., 75 (1998), 2698-2711.

[24] F. Rienzo, R. Gabdoulline, M. Menziani, P. Benedetti, R. Wade. Electrostatic analysis and brownian dynamics simulation of the association of plastocyanin and cytochrome $f$. Biophys. J., 81 (2001), 3090-3104.

[25] G.Y. Riznichenko, N.E. Belyaeva, I.B. Kovalenko, A.B. Rubin. Mathematical and computer modeling of primary photosynthetic processes. Biophys. J., 54 (2009), No. 1, 10-22.

[26] G.Y. Riznichenko, I.B. Kovalenko, A.M. Abaturova, A.N. Diakonova, D.M. Ustinin, E.A. Grachev, A.B. Rubin. New direct dynamic models of protein interactions coupled to photosynthetic electron transport reactions. Biophys. Rev., 2 (2010), No. 3, 101-110.

[27] A. Rubin, G. Riznichenko. Modeling of the primary processes in a photosynthetic membrane. Photosynthesis in silico: Understanding Complexity from Molecules to Ecosystems. (Eds. A. Laisk, L. Nedbal, and Govindjee). Springer, Dordrecht, 2009, 151-176.

[28] P. Setif. Electron transfer from the bound Iron-Sulfur clusters to Ferredoxin/Flavodoxin: Kinetic and structural properties of Ferredoxin/Flavodoxin reduction by photosystem I. In: Photosystem I: The Light-Driven Plastocyanin:Ferredoxin Oxidoreductase. (Ed. J.H. Golbeck). Springer, 2006, 439-454.

[29] K. Sigfridsson. Ionic strength and pH dependence of the reaction between plastocyanin and photosystem 1. Evidence of a rate-limiting conformational change. Photosynth. Res., 54 (1997), 143-153.

[30] M. Ubbink, M. Ejdebeck, B.G. Karlsson, D.S. Bendall. The structure of the complex of plastocyanin and cytochrome $f$, determined by paramagnetic NMR and restrained rigid-body molecular dynamics. Structure, 6 (1998), 323-335.

[31] G.M. Ullmann, E.-W. Knapp. Electrostatic models for computing protonation and redox equilibria in proteins. Eur. Biophys. J., 28 (1999), No. 7, 533-551.

[32] G.M. Ullmann, E.-W. Knapp, N.M. Kostic. Computational simulation and analysis of dynamic association between plastocyanin and cytochrome $f$. Consequences for the electrontransfer reaction. J. Amer. Chem. Soc., 119 (1997), 42-52. 